\title{
Evaluation of the Adhesive Interface of Resin Cements Using Scan- ning Electron Microscope
}

\author{
Ana Paula Gebert de Oliveira Franco ${ }^{1 *}$, Osnara Maria Mongruel Gomes ${ }^{2}$, Sérgio \\ Vieira ${ }^{3}$ and Rui Fernando Mazur ${ }^{3}$
}

${ }^{1}$ University Technological Federal of Paraná, Brazil

${ }^{2}$ University State of Ponta Grossa, Brazil

${ }^{3}$ Pontifical University Catholic of Paraná, Brazil

*Corresponding author: Ana Paula Gebert de Oliveira Franco, University Technological Federal of Paraná, Rua Francisco Rocha, 62. Sala 502. Batel, Curitiba, Paraná, Brazil, E-mail: anapaula.gebert@gmail.com

\begin{abstract}
Purpose: The objective of this study was to evaluate the presence of gaps between dentin and resin cement interface in different root thirds before and after application of a compressive load.

Methods: Sixty human premolars were extracted and endodontically treated and glass fiber translucent posts (White Post DC1) were cemented to the root canal using three luting systems: RelyX ARC; AllCem; and RelyX U-100. Thirty control teeth were not subjected to load application. The other half of the specimens were coronally reconstructed with composite resin (Filtek Z250) and subjected to a compressive force of $220 \mathrm{~N}$ at $1 \mathrm{~mm} / \mathrm{min}$. The adhesive dentin-resin cement interfaces of all specimens were evaluated using scanning electron microscopy. Measurements were obtained using Image Tool 3.0 software. The data were analyzed by two-way ANOVA and the Games-Howell test $(\alpha=$ 0.05 ). The percentage of adhesive interfaces in continuity was analyzed using a chi-squared test.
\end{abstract}

Results: The RelyX U100 cement had the highest adhesive continuity and lowest values for gap length $(p<0.05)$. The cervical third presented the lowest values gaps when compared with other root thirds $(p<0.05)$. There were no significant differences between the samples before or after the application of compressive load $(p<0.05)$.

Conclusion: Adhesive continuity and presence of gap were affected by the type of resin cement and the root third.

\section{Keywords}

Resin cements, Root dentin, Interface, Scanning electron microscope

\section{Introduction}

Root canal posts have been extensively used in the reconstruction of traumatized and decayed teeth and their function is to retain coronal restoration, with the goal of restoring lost dental structure [1]. Glass fiber posts have an elastic modulus closer to that of dentin, allowing a more uniform distribution of forces in the dental structures when compared to metal posts $[2,3]$. In addition, there is a discussion about the transmission of light through translucent posts to the polymerization of resin cements in deep portions of the root canal [4].

Adhesive cementation may reduce microleakage [5] and improve the retention of root posts [6]. Dual-cured cements have been developed to combine the favorable characteristics of self-curing and photo-activation [7], but they have incompatibility with all-in-one and twostep adhesive systems [8-10]. Dual resin cements have demonstrated adhesion capacity through the hybrid layer when used in association with total-etch adhesive systems [11,12]. Recently, self-adhesive cements, which do not require dentin pre-treatment, have been introduced to the market in an attempt to simplify the cementation procedure $[13,14]$. The adhesion mechanism of self-adhesive cements is based on the acidic monomers (esters of phosphoric acid) that simultaneously demineralize infiltrate the dentin surface [15], thereby creating micromechanical retention and chemical adhesion to hydroxyapatite [16]. However, some of

Citation: de Oliveira Franco APG, Gomes OMM, Vieira S, Mazur RF (2018) Evaluation of the Adhesive Interface of Resin Cements Using Scanning Electron Microscope. Int J Oral Dent Health 4:052. doi. org/10.23937/2469-5734/1510052

Received: March 10, 2016: Accepted: May 12, 2018: Published: May 14, 2018

Copyright: (C) 2018 de Oliveira Franco APG, et al. This is an open-access article distributed under the terms of the Creative Commons Attribution License, which permits unrestricted use, distribution, and reproduction in any medium, provided the original author and source are credited. 
them present a limitation concerning the high viscosity that requires application with higher pressure inside the root canal [17].

There are many factors that are considered challenges related to adhesion into root canal: Inadequate access [18], geometrical factors [19] and the peculiar histological features of the root dentin [20], the density and orientation of the dentinal tubules [21], presence of the endodontic smear layer [22], moisture control in the root canal [23], the technique-sensitivity of applying adhesive systems [8], and polymerization shrinkage (C-factor) [20].

The high and unfavorable C-factor of root canals, which occurs due to the large bonded surface area and the reduced free surface area, can contribute to maximizing the polymerization stress of resin-based materials [24-26] along the walls of root canals [27] and result in interfacial gaps [28]. Several factors influence the amount of tension generated during the polymerization contraction, such as the size, the quantity and the form of the filler particles that make up the polymer [29], the elasticity modulus [30], the volume of material, the rate of polymerization, and the gelation time [31]. Glass fiber post retention depends not only on obtaining adhesion free of gaps, but also on its frictional retention in the root canal to ensure greater stability [32,33]. Little is known about measurement of the size of gaps inside a root canal and the percentage of adhesive continuity between the dentin and the resin cement (D/C).

Thus, the aim of this study was to evaluate the presence of gaps between $D / C$ in different root thirds both before and after application of a static functional load. The hypothesis were that: 1) There would be significant difference among the resin cements regardless of the root thirds; 2) There would be significant differences between no loading and loading specimens.

\section{Methods}

\section{Preparation of specimens}

Selection of specimens: Sixty human lower premolars were selected from the tooth bank of the Catholic University of Parana (PUCPR), with the approval of the Research Ethics Committee of PUCPR, n. 2797. Exclusion criteria were the presence of severe curvature, root caries, structural defects of the enamel, and previous endodontic treatment. The average length of the selected teeth was $22.66 \mathrm{~mm}$.

Endodontic treatment: The canals of all the specimens were treated according to the crown-down technique and instrumented with nickel-titanium endodontic files (Dentsply, Maillefer, Weybridge, UK) until a diameter corresponding to the memory instrument ISO 40 was reached. The apical limit of the root canal treatment was determined to be $1 \mathrm{~mm}$ below the apical foramen. Five percent of sodium hypochlorite and $17 \%$
EDTA were used as endodontic irrigation agents, and the canals were dried with absorbent paper points (Dentsply, Petrópolis, RJ, Brazil). The root canals were filled by lateral condensation with main gutta-percha cones, gutta-percha accessory cones, and endodontic Sealer [26] (Dentsply). Lamination and vertical condensation of the gutta-percha cones were performed using the thermoplastic hybrid technique (McSpadden; Dentsply, Maillefer, Weybridge, UK). The pulp chamber was sealed with Coltosol temporary cement (Vigodent, S/A, Rio de Janeiro, RJ, Brazil). The specimens were stored in distilled water and kept at $37^{\circ} \mathrm{C}$ for 7 days.

Preparation of specimens for analysis of the adhesive interface: The coronary surfaces of each specimen were sectioned $2 \mathrm{~mm}$ above the cement-enamel junction (CEJ), perpendicular to the long axis, using a lowspeed diamond disc (Isomet 1000; Buehler, Illinois, USA) with water irrigation.

Sixty specimens were randomly divided into 3 groups of 20 teeth each:

- Group ARC: Adper Scotchbond Multi-purpose adhesive system and RelyX ARC dual resin cement (3M/ ESPE, St. Paul, MN, USA);

- Group ALLCEM: Adper Scotchbond Multipurpose adhesive system and AllCem dual resin cement (FGM, Joinville, SC, Brazil); and

- Group U100: RelyX U100 dual self-adhesive resin cement (3M/ESPE).

The materials used in this study are listed in Table 1.

Gutta-percha was partially removed with heated pluggers (Duflex, SS White, Rio de Janeiro, RJ, Brazil). The root canal of each specimen was enlarged with lowspeed drills (FGM) provided by the manufacturer of the post system. The depth of the intraradicular preparation was $10 \mathrm{~mm}$ of the CEJ, with a diameter equal to the space for the post, and was standardized for all teeth $[11,12]$. The cut of the White Post DC1 posts were performed after the preparation (FGM). The final irrigation was performed with a $5 \% \mathrm{NaOCl}$ solution, and the specimens in all groups were then dried with absorbent paper points (Dentsply).

The glass fiber posts were cleaned with $70 \%$ alcohol and underwent application of the RelyX ceramic primer silane agent (3M/ESPE). In Groups ARC ( $n=20)$ and ALLCEM $(n=20)$, the canals were etched with $35 \%$ phosphoric acid for 15 seconds, washed with distilled water for 15 seconds, and then dried for 5 seconds. The absorbent paper point was used to remove any excess water in the canal. Adper Scotchbond multi-purpose plus activator (3M/ESPE) was applied in the canal using a paper point and dried for 5 seconds. After, the primer was applied into the canal using a paper point and dried for 5 seconds and catalyst was applied inside the canal with paper point. A coating of Adper Scotchbond 
Table 1: Description of the materials used in the study.

\begin{tabular}{|c|c|c|c|c|}
\hline Material & Comercial brand & Composition & Manufacturer & Serial number \\
\hline Glass fiber post & White Post DC 1 & $\begin{array}{l}\text { Fiberglass, epoxy resin, inorganic fillers, silane, } \\
\text { promoters of polymerization. }\end{array}$ & FGM LTDA & 60709 \\
\hline Phosphoric Acid & Cond Ac 37 & $35 \%$ phosphoric acid and thickener. & FGM LTDA & F/L11 \\
\hline \multirow[t]{3}{*}{ Adhesive system } & $\begin{array}{l}\text { Adper Scotchbond } \\
\text { Multi-purpose }\end{array}$ & $\begin{array}{l}\text { Primer: water; 2-hydroxyethyl methacrylate; } \\
\text { copolymer of acrylic and itaconic acids (5AT). }\end{array}$ & 3M/ESPE & Primer 9CC \\
\hline & & $\begin{array}{l}\text { Activator: ethyl alcohol; sodium benzenesulfinate } \\
(5 \mathrm{KT}) \text {. }\end{array}$ & & Activator 9RL \\
\hline & & $\begin{array}{l}\text { Catalyst: bis-GMA; 2-hydroxyethyl } \\
\text { methacrylate; benzoyl peroxide (3AP) }\end{array}$ & & \\
\hline Silane & RelyX Ceramic Primer & Single-phase hydrolyzed solution. & 3M/ESPE & $9 A B$ \\
\hline Resin cement & RelyX ARC & $\begin{array}{l}\text { Paste A: } 68 \% \text { load particles (zirconia/silica), } \\
\text { pigments, amine and photoinitiator system. } \\
\text { Paste B: } 67 \% \text { filler, benzoyl peroxide. }\end{array}$ & 3M/ESPE & GX9JK \\
\hline \multirow[t]{2}{*}{ Resin cement } & RelyX U100 & $\begin{array}{l}\text { Base paste: fiberglass, phosphoric acid ester } \\
\text { methacrylate, triethylene glycol dimethacrylate, } \\
\text { silica. }\end{array}$ & 3M/ESPE & 343485 \\
\hline & & $\begin{array}{l}\text { Catalyst paste: fiberglass, dimethacrylate, silica } \\
\text { treated with silane, sodium p-toluenesulfonate } \\
\text { and calcium hydroxide. }\end{array}$ & & \\
\hline Resin cement & AllCem & $\begin{array}{l}\text { Base paste: TEGDMA, Bis EMA and BisGMA, } \\
\text { camphorquinone, co-initiators, micro-particles } \\
\text { of barium aluminosilicate glass, silicon } \\
\text { dioxide nanoparticles, inorganic pigments and } \\
\text { preservatives. Catalyst paste: methacrylic } \\
\text { monomers, dibenzoyl peroxide, stabilizers and } \\
\text { microparticles. }\end{array}$ & FGM LTDA & 131107 \\
\hline
\end{tabular}

Multi-purpose plus catalyst was applied to the post. The luting agent was applied to the root canal with a Centrix syringe (DFL, Rio de Janeiro, RJ, Brazil). The translucent glass fiber post was seated inside the root canal and stabilized for 3 minutes with digital pressure. The resin cement was photoactivated from the occlusal surface for 40 seconds at $600 \mathrm{~mW} / \mathrm{cm}^{2}$ (Demetron, Kerr, Optilux 500, Danbury, USA).

The U100 group ( $n=20)$, the root canal walls were lightly dried with absorbent paper points. The resin cement was applied to the root canals with a Centrix syringe. After the post was placed in the root canal, it was stabilized for 3 minutes with finger pressure, and the resin cement was photoactivated from the occlusal surface for 40 seconds at $600 \mathrm{~mW} / \mathrm{cm}^{2}$ (Demetron). All the samples were stored for 24 hours in distilled water at 37 ${ }^{\circ} \mathrm{C}$. Only half of the specimens were used for the analysis of the adhesive interface between D/Cs. The other specimens underwent a compressive load test.

Preparation of the specimens without load application: To evaluate the $\mathrm{D} / \mathrm{C}$ interface, the apical portions of the specimens were removed. Then, the specimens were longitudinally sectioned with an Isomet 1000 diamond saw (Buehler) at low speed and under constant irrigation. The sections were then sequentially polished with $600,800,1,000$, and 1,200 grit sandpaper (Norton Saint Gobain, Rio de Janeiro, RJ, Brazil) under water. The specimens underwent ultrasound (Dabi Atlante, Ribeirao Preto, SP, Brazil) for 10 minutes for residue removal. Then, epoxy resin replicas Alpha Resiqualy (Curitiba, PR, Brazil) were prepared from additional silicone impressions of tooth surfaces (Express XT, 3M/ESPE) for later SEM analysis.

Preparation of specimens subjected to a compressive load: The specimens were embedded in vinyl polychloride rings (20 $\mathrm{mm}$ in diameter and $14 \mathrm{~mm}$ in height) with acrylic resin (Jet, Clássico, São Paulo, SP, Brazil) to the CEJ. To simulate the presence of the periodontal ligament, a thin layer of addition-cured silicone (Express XT, 3M/ ESPE) was created. To perform the cervical preparation of the coronal portion of the tooth remaining, 1014, 4138, $4138 \mathrm{~F}$ and $4138 \mathrm{FF}$ dental diamond points (KG Sorensen, São Paulo, SP, Brazil) were used. In all the samples, 1-mm thick and 2-mm high full crown preparations were made. To obtain the coronal portion of the core, a mold was made of standard acetate with the aid of Nite White vacuum plasticizer (Nite White, Culver City, CA, USA) with an anatomical shape of the crown of the lower premolar.

For restoration of the crown, the remaining tooth structure was conditioned with $37 \%$ phosphoric acid (AC 37 Cond, FGM) for 15 seconds, rinsed with water for 15 seconds and dried with small cotton balls. Then, a layer of primer was applied to the moist dentin, and two layers of Adper Scotchbond Multi-purpose Adhesive (3M/ ESPE) were applied 20 seconds later. The surface was polymerized for 20 seconds with a halogen lamp at 600 $\mathrm{mW} / \mathrm{cm}^{2}$ (Demetron). Layers of 2-mm-thick composite resin (Filtek Z250; 3M/ESPE) were applied around the prefabricated post and photoactivated for 20 seconds each. For the crown fabrication, the acetate mold was filled with resin and photoactivated for 40 seconds. After curing, the acetate molds were removed. 

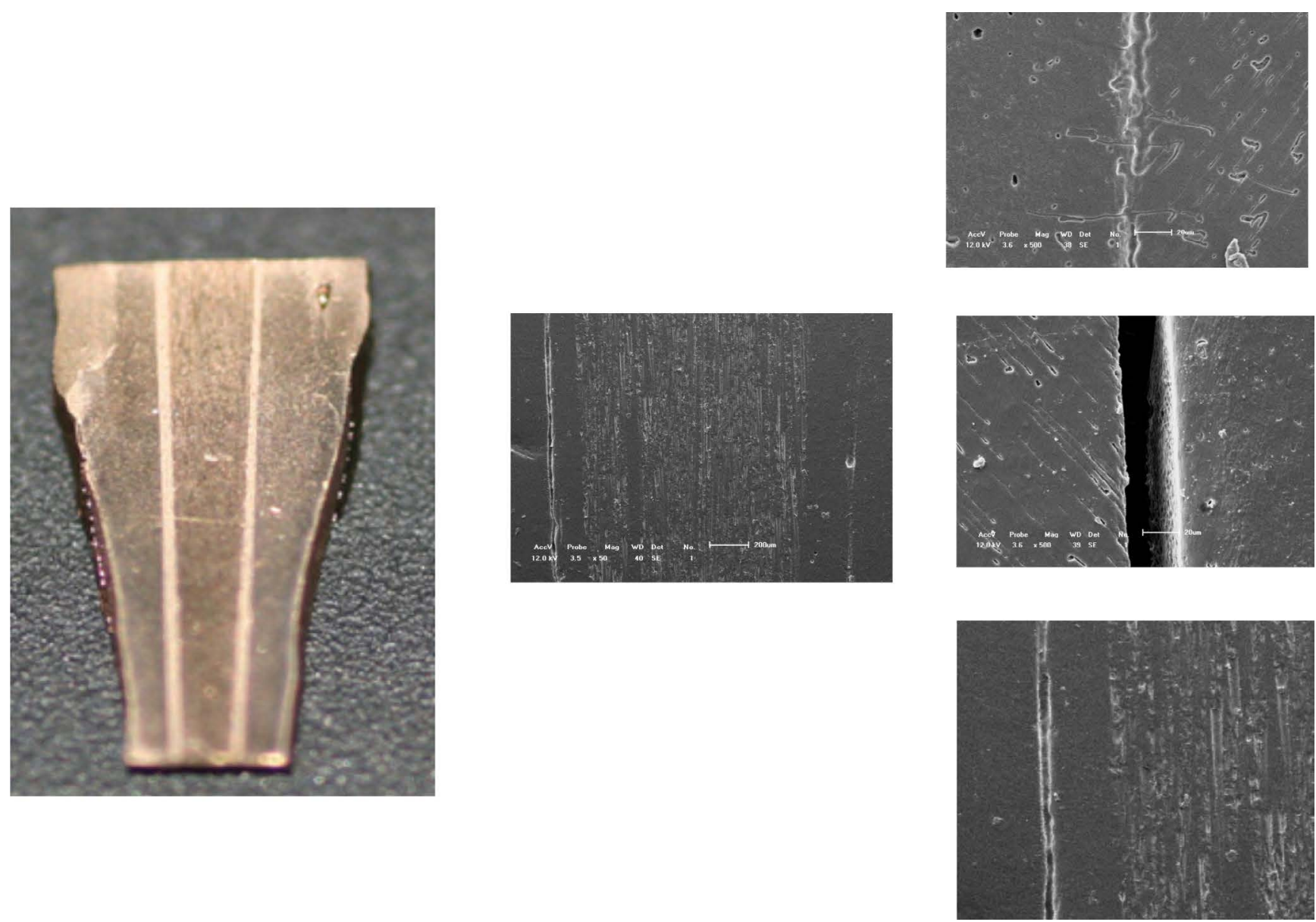

Figure 1: Image representing the electron microscopy scanning images A) A replica of the specimen section; B) The cervical third (50x); C) The region without gap in the interface dentin-resin cement (500x); D) The region demonstrating a gap at the dentin-resin cement interface (500x); and E) Gap at the dentin-resin cement interface (50x).

The specimens were subjected to a compression test in a universal testing machine (EMIC, São José dos Pinhais, PR, Brazil) with a device to standardize the inclination of the specimen at the base of the equipment. A force of $220 \mathrm{~N}$ was applied at $135^{\circ}$ to the buccal chewing surfaces of the lower premolars at a speed of $1 \mathrm{~mm} /$ $\min [3,34]$. The specimens were not fractured. After 24 hours of storage in distilled water at $37^{\circ} \mathrm{C}$, the specimens underwent the same procedures to obtain epoxy resin replicas for adhesive interface $D / C$ analysis.

Preparation of specimens for scanning electron microscope (SEM) and measurement of the cementing line and adhesive interface: Each replica was mounted on a metal stub, sputtered with gold (BAL-TEC SC_RD 005, BAL-TEC AG, Balzers, Liechtenstein) and observed under a scanning electron microscope (model SSX-550, Shimadzu, Kyoto, Japan) at different magnifications (Figure 1A). The adhesive interfaces between the dentin-cement-post in the cervical, middle and apical thirds of the mesial, and distal regions around the post were analyzed. First, an image with 50x magnification of each root third of the specimen was obtained (Figure 1B). Each image enabled the evaluation of the adhesive continuity or the presence of gaps. Next, the most representative area of the root third of the specimen for each side (mesial and distal) was selected, and another image with higher magnification (500x) was obtained. This second image confirmed the presence or absence of gaps (Figure 1C, Figure 1D and Figure 1E). The measurements were performed using Image Tool software version 3.0. The calibration of spatial measurements was carried out according to a numeric scale determined by SEM. The largest amplitude in each root third was used to evaluate the gaps. Each image was divided into 3 equal parts and 3 measurements were performed in each region.

\section{Statistical Analysis}

\section{Analysis of the teeth}

Kolmogorov-Smirnov test $(p<0.05)$ was used to verify the homogeneity of the teeth length. The average value was $22.66 \pm 1.09 \mathrm{~mm}$.

\section{Analysis of the adhesive interface between dentin and cement}

Levene's test for homogeneity of variance and a twoway ANOVA were used to measure the gaps in different root thirds with different resin cements $(p<0.05)$. Since the variances were heterogeneous, the Games-Howell multiple comparisons test was applied $(p<0.05)$. To assess the percentage of adhesive continuity, a chisquared test was used $(p<0.05)$. 
Table 2: Mean values (SD) of the gaps $(\mu \mathrm{m})$ for the resin cements in root thirds to no loading and loading specimens. Root Thirds

\begin{tabular}{|c|c|c|c|c|c|c|}
\hline \multirow[b]{2}{*}{$\begin{array}{l}\text { Loading } \\
\text { Conditions }\end{array}$} & \multicolumn{2}{|l|}{ Coronal } & \multicolumn{2}{|l|}{ Medium } & \multicolumn{2}{|l|}{ Apical } \\
\hline & No loading & Loading & No loading & Loading & No loading & Loading \\
\hline Resin cements & $(\mu \mathrm{m})$ & $(\mu \mathrm{m})$ & $(\mu \mathrm{m})$ & $(\mu \mathrm{m})$ & $(\mu \mathrm{m})$ & $(\mu \mathrm{m})$ \\
\hline RelyX ARC & $8.38(11.17)^{\mathrm{ABC}}$ & $5.57(7.29)^{\mathrm{A}}$ & $11.92(8.44)^{\mathrm{BE}}$ & $17.18(14.95)^{\mathrm{BCE}}$ & $12.02(11.98)^{\mathrm{BE}}$ & $14.79(18.74)^{\mathrm{BCE}}$ \\
\hline AllCem & $6.44(9.11)^{\mathrm{C}}$ & $9.44(21.28)^{\mathrm{ABCE}}$ & $11.56(15.03)^{\mathrm{BCE}}$ & $11.67(10.48)^{\mathrm{BE}}$ & $11.15(14.56)^{\mathrm{BCE}}$ & $16.35(16.36)^{\mathrm{E}}$ \\
\hline RelyX U100 & $4.64(8.17)^{\mathrm{ACD}}$ & $2.06(4.49)^{\mathrm{D}}$ & $7.08(9.11)^{\mathrm{ABC}}$ & $4.58(7.35)^{A D}$ & $8.19(13.10)^{\mathrm{ABC}}$ & $7.47(7.68)^{\mathrm{AC}}$ \\
\hline
\end{tabular}

Different capital letters indicate significant differences $(p \leq 0.05)$.

Table 3: Percentage values for adhesive continuity (\%) of the resin cements in root thirds to no loading and loading specimens.

\begin{tabular}{|l|l|l|l|l|l|l|}
\hline Root Thirds & \multicolumn{3}{l|}{ Cervical } & \multicolumn{3}{l|}{ Apical } \\
\hline Loading condition/Resin Cements & No loading & Loading & No loading & Loading & No loading \\
\hline & $(\%)$ & $(\%)$ & $(\%)$ & $(\%)$ & $(\%)$ & $(\%)$ \\
\hline RelyX ARC & $55^{\mathrm{A}}$ & $60^{\mathrm{B}}$ & $20^{\mathrm{B}}$ & $25^{\mathrm{B}}$ & $50^{\mathrm{B}}$ & $50^{\mathrm{A}}$ \\
\hline AllCem & $60^{\mathrm{A}}$ & $75^{\mathrm{AB}}$ & $45^{\mathrm{A}}$ & $35^{\mathrm{B}}$ & $40^{\mathrm{AB}}$ & $40^{\mathrm{A}}$ \\
\hline RelyX U100 & $65^{\mathrm{A}}$ & $80^{\mathrm{A}}$ & $55^{\mathrm{A}}$ & $65^{\mathrm{A}}$ & $60^{\mathrm{A}}$ & $45^{\mathrm{A}}$ \\
\hline
\end{tabular}

Different capital letters in the column indicate significant differences $(p \leq 0.05)$.

\section{Results}

The specimens subjected to a compressive load revealed no significant differences from the no loading specimens in analysis of variance (ANOVA) $(p<0.05)$. A comparison of cements in root thirds for the loading conditions revealed that the self-adhesive resin cement RelyX U100 showed the lowest gap values in the cervical third for loading specimens $(2.06 \pm 4.49 \mu \mathrm{m})$, and that the dual resin cement RelyX ARC demonstrated the highest values in the middle third for loading specimens (17.18 $\pm 14.95 \mu \mathrm{m})$ (Table 2).

The loading specimens of dual self-adhesive resin cement RelyX U100 used in the cervical third revealed the highest percentage of adhesive continuity (80\%). RelyX ARC demonstrated the lowest values $(20 \%)$ in the middle third to no loading specimens (Table 3 ).

Considering the sample size, was calculated the power of analysis when applying the method two-way ANOVA showed a $99.99 \%$ power to accept the hypothesis statistically significant difference between cement and root thirds. There was a low power to accept the hypothesis statistically difference between loading and no loading specimens, confirming the results obtained, since the ANOVA identified no statistically significant differences between the loading and no loading specimens $(p=0.2566)$. Thus the sample size used is representative for accepting the hypothesis region difference between cement and root thirds, since the power was in both cases above $99 \%$.

\section{Discussion}

The first hypothesis of this study was accepted because significant differences between resin cements and root thirds were observed. The gap values found at the $D / C$ interface suggesting that adhesion was better in the cervical third. This finding is consistent with a study by Bonfante, et al. [18].
In addition to inadequate access to the deeper portions of the root canal, there are other clinical challenges related to adhesion to intraradicular dentin, such as the peculiar histological features of the root dentin [20], the density and orientation of the dentinal tubules [21], presence of the endodontic smear layer [22], moisture control in the root canal [23], the sensitive technique for applying adhesive systems 8 , and polymerization shrinkage (C-factor) [20].

The contraction that occurs during polymerization of composite resins is approximately 1.5 to $5 \%$, which results in the development of internal tensions [25]. These stresses are transferred to the interface in the form of tensile forces [26], and the greater the constriction of the area to be filled, the greater the magnitude of the resulting strains. The gaps found in this study were the result of polymerization shrinkage forces that exceeded the adhesion strength of the cementing system to the root dentin $[18,19,27]$.

Several factors influence the amount of tension generated during the polymerization contraction, such as the size, the quantity and form of the filler particles that make up the polymer [29], the elasticity modulus [30], the volume of material, the rate of polymerization, and the gelation time [31]. Furthermore, additional tensions can arise with clinical use of the material due to its thermal expansion and contraction.

Despite the presence of gaps in the adhesive interface between $D / C$, there were areas where the integrity of the interface was maintained. This integrity can be measured by the percentage of adhesive continuity between $D / C$. In the present study, the percentage of adhesive continuity between $\mathrm{D} / \mathrm{C}$ depended on the resin cement used and the root third. The high percentage of continuity of the interface and the lowest mean values of gaps found for the RelyX U100 suggest that 
this resin cement has better adhesion to the root canal than the other resin cements investigated in this study. The highest value found was $80 \%$ for RelyX U100 at the cervical third after load application, which contradicts a report by Bonfante, et al. [18] of a $30 \%$ maximum adhesive continuity for all groups of resin cements studied. All the resin cements used in this study showed a higher percentage of adhesive continuity at the cervical third. These results are consistent with those of Radovic, et al. [4]. Boing, et al. [35] and Bazzo, et al. [36] showed lower bond strength in apical third compared to other root regions.

The self-adhesive cements present micromechanical retention generated by dental demineralization and simultaneous infiltration by methacrylate monomers, and chemical adhesion to hydroxyapatite [16,37], which may have influenced the higher percentage of adhesive continuity observed in this study. However, RelyX U100 has a limited potential for conditioning when compared to cementing systems that use conventional three-step adhesives [15] Giachetti, et al. [33] reported that the retention of RelyX U100 to root dentin appeared to be related more to friction along the walls of the root canal than to dentin bonding, which supports the limited potential for conditioning of RelyX U100. Sarkis-Onofre, et al. [37] in their systematic review concluded that the self-adherence, and dual-cure mechanism of the self-adhesive resin cements seems to improve the bonding of glass fiber posts into the confines of the root canal when compared with the conventional resin cements. The high viscosity of this material is a limitation that requires application with higher pressure inside the root canal [17].

The dual resin cements used in this study combine the favorable properties of self-polymerizable and photopolymerizable systems, ensuring polymerization without the influence of depth, enough time for photoactivation, and control of the setting reaction [7]. Their major disadvantage is technical sensitivity, due to the number of steps, because these procedures do not present a homogeneous adhesion line [9]. The dual resinous cement systems were used in association with the Adper Scotchbond Multi-purpose Plus (3M/ESPE) adhesion system, a three-step application described as the gold standard for polymer adhesion. This type of adhesive system is compatible with dual resin cements and allows for deeper penetration by demineralization with $37 \%$ phosphoric acid, which favors micromechanical imbrication [12]. This is contrast to the conventional two-step and one-step self-etching adhesive systems that contain acidic monomers that result in a slow conversion of the tertiary amine, thereby promoting the inhibition of benzoyl peroxide activation and free radical formation responsible for initiating the chemical polymerization reaction of the cement in the absence of a light source [10].
None of the samples tested in this study showed defects in the cement/post bonding, but adhesive defects in the $D / C$ interface was observed, which is consistent with previous studies $[17,23]$. Glass fiber post retention depends not only on obtaining adhesion free of gaps, but also on its frictional retention in the root canal to ensure greater stability [32].

Future studies should test the dynamic forces that can better represent intraoral chewing, replicate thermal cycling with different temperatures and perform a 3D analysis of the adhesive interface with micro-CT.

\section{Conclusions}

Within the limitations of this study, the following can be concluded:

1. RelyX U100 showed greater continuity of the adhesive interface and lower values of gaps than RelyX ARC or AllCem;

2. The cervical third revealed less gap formation, and the lowest values for RelyX U100;

3. No loading specimens demonstrated no significant differences in mean gap values or continuity of the adhesion interface between $\mathrm{D} / \mathrm{C}$ when compared to loading specimens.

\section{Conflicts of Interest}

Absent.

\section{References}

1. Assif D, Gorfil C (1994) Biomechanical consideration in restoring endodontically treated teeth. J Prosthet Dent 71 : 565-567.

2. Asmussen E, Peutzfeld A, Heitmann T (1999) Stiffness, elastic limit, and strength of newer types of endodontic posts. J Dent 27: 275-278.

3. Wakabayashi N, Ona M, Suzuki T, Igarashi Y (2008) Nonlinear finite element analyses: Advances and challenges in dental applications. J Dent 36: 463-471.

4. Radovic I, Corciolani G, Magni E, Krstanovic G, Pavlovic V, et al. (2009) Light transmission through fiber post: The effect on adhesion, elastic modulus and hardness of dual-cure resin cement. Dent Mater 25: 837-844.

5. Reid LC, Kazemi RB, Meiers JC (2003) Effect of fatigue testing on core integrity and post microleakage of teeth restored with different post systems. J Endod 29: 125-131.

6. Hedlund SO, Johansson NG, Sjogren G (2003) Retention of prefabricated and individually cast root canal posts in vitro. Br Dent J 195: 155-158.

7. Perdigão J, Geraldeli S, Lee IK (2004) Push-out bond strengths of tooth-colored posts bonded with different adhesive systems. Am J Dent 17: 422-426.

8. Van Meerbeek B, Van Landuyt K, De Munck J, Hashimoto M, Peumans M, et al. (2005) Technique-sensitivity of contemporary adhesives. Dent Mater J 24: 1-13.

9. Bandéca MC, Kassem AS, Nadalin MR, Queiroz RS, Clavijo VRG, et al. (2010) Influence of storage times on bond strength of resin cements to root canal. Mater Res 13: 25-28. 
10. De Santis R, Mollica F, Prisco D, Rengo S, Ambrosio L, et al. (2005) A 3D analysis of mechanically stressed dentin-adhesive-composite interfaces using X-ray micro-CT. Biomaterials 26: 257-270.

11. Nakabayashi N, Kojima K, Masuhara E (1982) The promotion of adhesion by infiltration of monomers into tooth substrates. J Biomed Mat Res 16: 265-273.

12. Noirrit EE, Grégoire G, Cournot M (2008) Morphological study of fiber-reinforced post-bonding system-root dentin interface by evaluation of two bonding systems. J Dent 36 : 204-213.

13. De Munck J, Vargas M, Van Landuyt K, Hikita K, Lambrechts $\mathrm{P}$, et al. (2004) Bonding of an auto-adhesive luting material to enamel and dentin. Dent Mater 20: 963-971.

14. Hikita K, Van Meerbeek B, De Munck J, Ikeda T, Van Landuyt K, et al. (2007) Bonding effectiveness of adhesive luting agents to enamel and dentin. Dent Mater 23: 71-80.

15. Behr M, Rosentritt M, Regnet T, Lang R, Handel G (2004) Marginal adaptation in dentin of a self-adhesive universal resin cement compared with well-tried systems. Dent Mater 20: 191-197.

16. Gerth HU, Dammaschke T, Züchner H, Schäfer E (2006) Chemical analysis and bonding reaction of RelyX Unicem and Bifix composites-A comparative study. Dent Mater 22 934-941.

17. Zicari F, Couthino E, De Munck J, Poitevin A, Scotti R, et al. (2008) Bonding effectiveness and sealing ability of fiber-post bonding. Dent Mater 24: 967-977.

18. Bonfante EA, Pegorarao LF, Góes MF, Carvalho RM (2008) SEM observation of the bond integrity of fiber-reinforced composite posts cemented into root canals. Dent Mater 24: 483-491.

19. Tay FR, Loushine RJ, Lambrechts P, Weller RN, Pashley $\mathrm{DH}$ (2005) Geometric factors affecting dentin bonding in the root canals: A theoretical modelling approach. J Endod 31: 584-589.

20. Ferrari M, Mannocci F, Vichi A, Cagidiaco MC, Mjör IA (2000) Bonding to root canal: Structural characteristics of the substrate. Am J Dent 13: 255-260.

21. Goracci C, Tavares AU, Fabianelli A, Monticelli A, Rafaelli O, et al. (2004) The adhesion between fiber posts and root canal walls: Comparison between microtensile and push-out bond strength measurements. Eur J Oral Sci 112: 353-361.

22. Vicenzi V, Plotino G, Giansiracusa A, Pietrangeli E, Sudani DA, et al. (2011) A SEM study of canal cleanliness after a new nickel-titanium rotatory instrumentation technique. Ann Stomatol 2: 19-22.

23. Bitter K, Paris S, Pfuertner C, Naumann K, Kielbassa AM (2009) Morphological and bond strength evaluation of different resin cements to root dentin. Eur J Oral Sci 177: 326333.
24. Feilzer AJ, De Gee AJ, Davidson CL (1987) Setting stress in composite resin in relation to configuration of the restoration. J Dent Res 66: 1636-1639.

25. Ferracane JL (2005) Developing a more complete understanding of stresses produced in dental composites during polymerization. Dent Mater 21: 36-42.

26. Carvalho RM, Pereira JC, Yoshiyama M, Pashley DH (1996) A review of polymerization contraction: The influence of stress development versus stress relief. Oper Dent 21: 17-24.

27. Prisco D, De Santis R, Mollica F, Ambrosio L, Rengo S, et al. (2003) Fiber post adhesion to resin luting cements in the restoration of endodontically-treated teeth. Oper Dent 28: 510-521.

28. Mota CS, Demarco FF, Camacho B, Powers JM (2003) Tensile bond strength of four resin luting agents bonded to bovine enamel and dentin. J Prosthet Dent 89: 558-564.

29. Satterthwaite JD, Vogel K, Watts DC (2009) Effect of resin-composite filler particle size and shape on shrinkage-strain. Dent Mater 25: 1612-1615.

30. Alster D, Feilzer AJ, Gee AJ, Mol A, Davidson CL (1992) The dependence of shrinkage stress reduction on porosity concentration in thin resin layers. J Dent Res 71: 16191622.

31. Stannsbury JW, Trujillo-Lemon M, Lu H, Ding X, Lin Y, et al. (2005) Conversion-dependent shrinkage stress and strain in dental resins and composites. Dent Mater 21: 56-67.

32. Goracci C, Fabianelli A, Sadek FT, Papacchini F, Tay FR, et al. (2005) The contribution of friction to the dislocation resistance of bonded fiber posts. J Endod 31: 608-612.

33. Giachetti L, Grandini S, Calamai P, Fantini G, Russo DS (2009) Translucent fiber post cementation using light- and dual-curing adhesive techniques and self-adhesive material: Push-out test. J Dent 37: 638-642.

34. Cimini Junior CA, Gouvêa P, de Las Casas EB, Cornacchia TPM (2000) Revisão crítica das medidas e modelos de cargas atuantes em dentes. $21^{\text {st }}$ Iberian Latin American Congress on Computacional Methods in engineering, Rio de Janeiro, RJ.

35. Sarkis-Onofre R, Skupien JA, Cenci MS, Moraes RR, Pereira-Cenci T (2014) The role of resin cement on bond strength of glass-fiber posts luted into root canals: A systematic review and metaanalysis of In Vitro Studies. Oper Dent 39: E31-E44.

36. Boing TF, Gomes GM, Gomes JC, Reis A, Gomes OM (2017) Is the bonding of self-adhesive cement sensitive to root region and curing mode? J Appl Oral Sci 25: 2-9.

37. Bazzo JF, Pedriali MB, Guiraldo RD, Berger SB, Moura SK, et al. (2016) Push-out bond strength of different translucent fiber posts cemented with self-adhesive resincement. J Conserv Dent 19: 583-586. 